\title{
Escape of atmospheric gases from the Moon
}

\author{
DA DAO-AN ${ }^{1, *}$ and YANG YA-TIAN ${ }^{2}$ \\ ${ }^{1}$ Lanzhou Institute of Physics, 730000 Lanzhou, Gansu, China. \\ ${ }^{2}$ Fujian Normal University, 350007 Fuzhou, Fujian, China. \\ *e-mail:dadaoan@sohu.com
}

The escape rate of atmospheric molecules on the Moon is calculated. Based on the assumption that the rates of emission and escape of gases attain equilibrium, the ratio of molecular number densities during day and night, $n_{0 d} / n_{0 n}$, can be explained. The plausible emission rate of helium and radioactive elements present in the Moon has also been calculated.

\section{Introduction}

A thick atmosphere is present on the Earth and Venus but it is only a very tenuous atmosphere, with a number density of $n_{0 d}=3 \times 10^{9} \mathrm{~m}^{-3}$ during day and $n_{0 n}=6 \times 10^{10} \mathrm{~m}^{-3}$ during night, consisting mainly of helium and argon which exist on the Moon. Whether a thick atmosphere existed on the Moon in the past or not is related to the process of formation of the Moon. One view (Konpaneetz 1957) is that the Moon did have a thick atmosphere in the beginning but owing to its weak gravity, the atmospheric molecules gradually escaped, resulting in the very thin atmosphere existing now. To see if a thick atmosphere could be retained or not, we assume that the air having pressure of 1 atmosphere (as exists on Earth now) existed on the Moon initially and calculate the escape life times for various assumed temperature distributions. The results show that if the atmospheric pressure is equal to or less than $1 \mathrm{~atm}$, the escape time is much less than the age of the Moon $\left(\sim 4.5 \times 10^{9} y\right)$, except for one special case, implying that any atmosphere $(p \leq 1 \mathrm{~atm})$ on the Moon would almost totally escape. These calculations also explain the observed day to night ratio, $n_{0 d} / n_{0 n} \approx 0.05$ based on the assumption that the emission rate of gases (mainly helium) from the Moon and the escape rate attain dynamic equilibrium. The emission rate on the surface of the Moon is estimated to be about $10^{23}$ molecules per second.

\section{Expression of escaping lifetime of atmosphere}

We derive an escape equation of atmosphere

$$
\frac{d N}{d t}=-\lambda N
$$

where $N$ is the total number of molecules in the atmosphere and $\lambda$ is the escape rate. The solution of (1) satisfying the initial condition of $N=N_{0}$ when $t=0$ is

$$
N=N_{0} e^{-\lambda t}
$$

When $t=1 / \lambda$

$$
N=N_{0} e^{-1}
$$

The escape life time is defined as

$$
\tau=\frac{1}{\lambda}
$$

Keywords. Escape rate; lunar atmosphere; radioactivity; helium. 
The expression of $\lambda$ with uniform temperature $T$ of the atmosphere is given as

$$
\lambda=\frac{\bar{v}}{4} f\left(\eta_{b}\right) \frac{G m M}{k T r_{b}^{2}} \exp \left\{\frac{G m M}{k T}\left(\frac{1}{r_{b}}-\frac{1}{r_{0}}\right)\right\} .
$$

Here $G$ is the gravitational constant, $m$ is the molecular mass of gas, $M$ is the mass of the planet, $\bar{v}=\sqrt{8 k T / \pi m}$ is the mean velocity of gas molecules, $k$ is the Boltzmann constant and $r_{0}$ is the effective radius of the planet. The function $f\left(\eta_{b}\right)$ is the percentage of number density with speed $v$ greater than the escape velocity. $r_{b}$ is radius of 'escape border' through which the molecules with velocity $v$ greater than escape velocity $v_{e}\left(v \geq v_{e}\right)$ can escape to space without collisions and is determined by the following condition:

$$
r \geq r_{b} \rightarrow \bar{l}(r) \geq \bar{h}(r)
$$

$\bar{l}(r)$ is the mean free path of molecules at $r . \bar{h}(r)$ is decided by:

$$
\frac{n(r+\bar{h}(r))}{n(r)}=\frac{1}{10}
$$

Here $n(r)$ is the molecular number density at a point $r$. The escape velocity $v_{e}=\sqrt{(2 G M / r)}$. Function $f(\eta)$ is the percentage of number density with speed $v$ greater than the escape velocity at the point $r$ over the total molecular number density at that point:

$$
\begin{aligned}
f(\eta) & \equiv \frac{n\left(v \geq v_{e}\right)}{n(v \geq o)}=\frac{2}{\sqrt{\pi}} \eta e^{-\eta^{2}}+1-\operatorname{erf}(\eta) \\
& \cong \frac{2}{\sqrt{\pi}} e^{-\eta^{2}}\left(\eta+\frac{1}{2 \eta}\right)
\end{aligned}
$$

$\eta \equiv v_{e}(r) / v_{p}, v_{p} \equiv \sqrt{(2 k T / m)}$. $\operatorname{erf}(x)$ is the error function. The approximate value of $f(\eta)$ is less than $0.1 \%$ if $\eta \geq 3$. We can see that $\eta$ is a function of $T$ and $v_{e}$ at $r$, and $\eta_{b}$ denotes the value of $\eta$ at $r=r_{b}$.

The temperature distribution of atmosphere mainly depends on the pressure (or density) of the atmosphere on a planetary body. If the atmosphere is thick like that on Earth, Venus and some satellites on Saturn, the temperature distribution is non-uniform; it varies with height and, for simplicity, we can divide the whole atmosphere into several 'isothermal regions' by introducing an effective temperature $T_{e}$ for each region to fit the measured data of pressure. That means $T=T_{e i}$ is a constant within the region $r_{i} \leq r<r_{i+1}$, $i=1,2, \ldots, q, q+1, \ldots, I$ as $r_{I+1} \rightarrow \infty$.

With this approximation, we obtain the following general expression for $\lambda$ :

$$
\begin{aligned}
& \lambda=\frac{\bar{v}}{4} f\left(\eta_{b}\right) \frac{G m M}{k T r_{b}^{2}} \\
& \quad\left\{\begin{array}{c}
\exp \left\{-\sum_{i=0}^{q-1} \frac{G m M}{k T_{e i}}\left(\frac{1}{r_{i}}-\frac{1}{r_{i+1}}\right)\right\} \\
\times \exp \left\{-\frac{G m M}{k T_{e q}}\left(\frac{1}{r_{q}}-\frac{1}{r_{b}}\right)\right\}
\end{array}\right\} \\
& 1-\exp \left\{-\sum_{i=0}^{I} \frac{G m M}{k T_{e i}}\left(\frac{1}{r_{i}}-\frac{1}{r_{i+1}}\right)\right\}
\end{aligned}
$$

Here $T$ is the real temperature at $r_{b}$. The escape border is within the isothermal region $r_{q} \leq r_{b}<r_{q+1}$, in which the effective temperature is $T_{e q}$. The denominator of the last term in (3A) originates from the normalization of number density:

$$
\int_{0}^{\infty} n(r) 4 \pi r^{2} d r=N .
$$

It should be summed over the whole region, taking $T_{e I}=T_{\infty}$ in the region of $r_{I} \leq r<r_{I+1}(=\infty)$ and summing up to $I$.

The following assumptions have been used to derive the above formula

- the temperature distribution does not change with time

- the escaping process of gas is a quasi-static process

- the molecular number density distribution of gas is given by a convergent formula, discussed elsewhere (Da Dao-an and Yang Ya-tian 2005).

\section{Escape rate of atmosphere on Moon}

The effective radius $r_{0}$, mass $M$, temperature during day and night: $T_{d}$ and $T_{n}$, acceleration of gravity $g$ of Moon are taken as follows:

$$
\begin{aligned}
r_{0} & =1738 \mathrm{~km}, \quad M=7.36 \times 10^{22} \mathrm{~kg}, \quad T_{d}=400 \mathrm{~K}, \\
T_{n} & =90 \mathrm{~K}, \quad g=1.62 \mathrm{~ms}^{-2} .
\end{aligned}
$$

In order to compare the retention ability of atmosphere on the Moon with Earth, we assume that the initial atmosphere of the Moon consisted of 'fictitious' molecules having a mean molecular weight of 28.8, and the initial pressure of 
Table 1. Escape rate $\lambda$ and life time $\tau$ for the assumed temperature distribution in the atmosphere on Moon.

\begin{tabular}{|c|c|c|c|c|c|c|c|c|c|}
\hline $\begin{array}{c}\text { Assumed } \\
\text { temperature } \\
\text { distribution }\end{array}$ & $\begin{array}{c}r_{b} \\
(\mathrm{~km})\end{array}$ & $\begin{array}{l}\bar{l}\left(r_{b}\right) \\
(\mathrm{km})\end{array}$ & $\begin{array}{l}\bar{h}\left(r_{b}\right) \\
(\mathrm{km})\end{array}$ & $\begin{array}{c}T\left(r_{b}\right) \\
(\mathrm{K})\end{array}$ & $(\mathrm{m} / \mathrm{s})$ & $\eta_{b}$ & $f\left(\eta_{b}\right)$ & $\begin{array}{c}\lambda \\
\left(\mathrm{s}^{-1}\right)\end{array}$ & $\begin{array}{c}\tau \\
(\mathrm{y})\end{array}$ \\
\hline (i) & $2.6 r_{0}$ & 794 & 695 & 240 & 104.6 & 3.98 & $6.11 \times 10^{-7}$ & $2.3 \times 10^{-21}$ & $1.4 \times 10^{13}$ \\
\hline (ii) & $8.5 r_{0}$ & $4.57 \times 10^{3}$ & $4.57 \times 10^{3}$ & 400 & 135.0 & 1.70 & 0.1228 & $1.2 \times 10^{-15}$ & $\begin{array}{r}2.6 \times 10^{7} \\
2\end{array}$ \\
\hline \multirow[t]{2}{*}{ (iii) } & $8.5 r_{0}$ & $4.57 \times 10^{3}$ & $4.57 \times 10^{3}$ & d: 400 & 135.0 & 1.70 & 0.1228 & $\lambda_{d}=1.2 \times 10^{-15}$ & $\begin{aligned} \tau & =\overline{\lambda_{d}+\lambda_{n}} \\
& =5.2 \times 10^{7}\end{aligned}$ \\
\hline & $1.32 r_{0}$ & $\begin{array}{l}69.0 \\
>27 \times 10^{5}\end{array}$ & $\begin{array}{l}60.8 \\
5.7 \times 10^{4}\end{array}$ & n: 90 & $\begin{array}{l}64.0 \\
270\end{array}$ & 9.12 & $\begin{array}{l}7.9 \times 10^{-36} \\
0.80\end{array}$ & $\begin{array}{l}\lambda_{n}=6.1 \times 10^{-50} \\
7.16 \times 10^{-15}\end{array}$ & \\
\hline (iv) & $47 r_{0}$ & $\geq 2.7 \times 10^{5}$ & $5.7 \times 10^{4}$ & 1600 & 270 & 0.725 & 0.789 & $7.16 \times 10^{-10}$ & $4.4 \times 10^{\circ}$ \\
\hline
\end{tabular}

the atmosphere was $1 \mathrm{~atm}$. One can calculate the escape rate by means of the formula (3) and (3A). Since we do not know the temperature distribution on the ancient Moon, in order to calculate the escape rate and life time, we assume four simple cases of temperature distributions:

(i) $T=240 \mathrm{~K}$, uniform temperature (= mean temperature of Earth's atmosphere),

(ii) $T=400 \mathrm{~K}$, uniform temperature (surface temperature of Moon at day),

(iii) $T_{d}=400 \mathrm{~K}, T_{n}=90 \mathrm{~K}$, surface temperature of Moon during day and night, respectively,

(iv) $T_{e 1}=240 \mathrm{~K}\left(r<(3 / 2) r_{0}\right)$,

$T=800 \mathrm{~K}\left(r=(3 / 2) r_{0}\right)$,

$T_{e 2}=1600 \mathrm{~K}\left(r>(3 / 2) r_{0}\right)$

similar to the temperature distribution on Earth during the solar active period. We simply suppose that the temperature distribution can be divided into two effective isothermal regions: (1) $r_{0} \leq r<(3 / 2) r_{0}$, in which the temperature is constant $\left(T_{e 1}=240 \mathrm{~K}\right)$, like the average temperature of the atmosphere on Earth below $80 \mathrm{~km} ;(\mathbf{2})$ the second effective isothermal region is $(3 / 2) r_{0}<r<\infty$, with constant temperature $T_{e 2}=1600 \mathrm{~K}$, like the temperature on Earth; (3) in the region of $(3 / 2) r_{0}-\varepsilon<r<(3 / 2) r_{0}+\varepsilon, \varepsilon \rightarrow 0$, we assume that the temperature varies continuously from $T_{e 1}$, suddenly increasing to $T_{e 2}$, through the point of $T=800 \mathrm{~K}$ during $r=(3 / 2) r_{0}$. We take $d=2 \times 10^{-10} \mathrm{~m}$ to calculate the mean free path of molecules. The results are shown in table 1 .

We calculate the numerical data from (i) to (iii) with formula (3), and (iv) by (3A). One can see from table 1 that the conditions $r \geq r_{b} \rightarrow \bar{l}(r) \geq$ $\bar{h}(r)$ are satisfied: (i) $794 \mathrm{~km}>695 \mathrm{~km}$; (ii) $4.57 \times$ $10^{3} \mathrm{~km}=4.57 \times 10^{3} \mathrm{~km}$; (iii) $4.57 \times 10^{3} \mathrm{~km}=$ $4.57 \times 10^{3} \mathrm{~km}$, and $69.0 \mathrm{~km}>60.8 \mathrm{~km}$; (iv) $2.7 \times$ $10^{5} \mathrm{~km}>5.7 \times 10^{4} \mathrm{~km}$. In case (iii) $\tau=2 /$ $\left(\lambda_{d}+\lambda_{n}\right)$, based on $N=N_{0} \exp \left\{-\lambda_{d} t / 2-\lambda_{n} t / 2\right\}$, but the difference of $\lambda$ between day and night is about 35 orders of magnitude, thus $\tau=2 /\left(\lambda_{d}+\lambda_{n}\right) \approx 2 / \lambda_{d}$ and the life time in case (iii) is twice the life time of case (ii) owing to almost no escape at night time. Taking the age of the Moon as $t_{M}=4.5 \times 10^{9} \mathrm{y}$, it is shown from the calculations that $100 \tau \leq t_{M}$, for all the three cases except (i). In case (i), $\tau=1.4 \times 10^{13} \mathrm{y}$, much greater than $t_{M}$ and only a small fraction of molecules would escape. Therefore, the atmosphere on the Moon would continue to exist with pressure near $1 \mathrm{~atm}$. But in real case, the higher the height, the higher the temperature would be for the thick atmosphere, until it approaches $T_{\infty}$ which is higher than $1000 \mathrm{~K}$, depending on the solar activity. During quiet periods, $T_{\infty}=\sim 1000 \mathrm{~K}$ and during solar active periods, $T_{\infty}=\sim 1600 \mathrm{~K}$. From the viewpoint of comparing the retention ability of atmosphere on the Moon with Earth, the condition of case (iv) is closer to the situation of Earth during the solar active period. In this case,

$$
\begin{aligned}
\tau & =1.8 \times 10^{6} y, \\
t_{M} & =4.5 \times 10^{9} y=2.5 \times 10^{3} \tau, \\
N & =N_{0} e^{-2.5 \times 10^{3}}=N_{0} \times 10^{-1.086 \times 10^{3}} .
\end{aligned}
$$

It is shown from these results that the atmosphere of the Moon, if it existed a long time ago, could not be retained for more than $10^{8} y$ and must have totally escaped.

\section{Molecular number densities on Moon during day and night}

Assuming that the distribution law of molecular number density is valid for the Moon, then one obtains

$$
\frac{n_{0}\left(T_{1}\right)}{n_{0}\left(T_{2}\right)}=\frac{T_{2}}{T_{1}}
$$


Here $n_{0}$ is the molecular number density on the surface of the Moon. Taking $T_{d}=400 \mathrm{~K}$, $T_{n}=90 \mathrm{~K}$,

$$
\frac{n_{0 d}}{n_{0 n}}=\frac{T_{n}}{T_{d}}=\frac{90}{400} \cong \frac{1}{4.44}=0.225 .
$$

But the measured value is $n_{0 d}=3 \times 10^{9} \mathrm{~m}^{-3}$, $n_{0 n}=6 \times 10^{10} \mathrm{~m}^{-3}$,

$$
\frac{n_{d}}{n_{0 n}}=\frac{1}{20}=0.05 .
$$

The measured ratio is only $1 / 4.5$ of the theoretical value. For the molecular number density of $n_{0}=3 \times 10^{9} \mathrm{~m}^{-3}-6 \times 10^{10} \mathrm{~m}^{-3}$ as on the Moon, the mean free path of molecules is very large, $\bar{l}=1 \times 10^{4} \mathrm{~km}-1.9 \times 10^{5} \mathrm{~km}$, so the molecules with $v \geq v_{e}$ on the surface of the Moon can escape. The energy of $\alpha$ particles emitted from thorium and uranium series elements is greater than $4 \mathrm{Mev}$, which corresponds to velocities greater than $1.4 \times 10^{4} \mathrm{~km} / \mathrm{s}$, much greater than the escape velocity $(\sim 2.4 \mathrm{~km} / \mathrm{s})$ on the Moon. Taking into account the energy losses while penetrating the rocks or soil of the Moon, the velocity distribution reduces to Maxwellian distribution, then equation (3) or $(3 \mathrm{~A})$ is valid. The measurements show that gases on the Moon are mainly composed of helium. It is believed that the amount of gas is the result of competition between the emission and escape processes of gases. Suppose the atmospheric molecular number on the Moon is $N$ and the emission particle number from lunar surface is $N_{r}$, then we have

$$
\left\{\begin{array}{l}
\frac{d N_{r}}{d t}=-\lambda_{r} N_{r}, \\
\frac{d N}{d t}=\lambda_{r} N_{r}-\lambda_{d} N \quad \text { (day), } \\
\frac{d N}{d t}=\lambda_{r} N_{r}-\lambda_{n} N \quad \text { (night). }
\end{array}\right.
$$

$\lambda_{r}$ is an equivalent decay constant of various radioactive series, $\lambda_{d}$ and $\lambda_{n}$ are the escape rates of gas during day and night respectively, the escape border is taken as the surface of the Moon, i.e., $r_{b}=r_{0}$, thus (3) is simplified as

$$
\lambda=\frac{\bar{v}}{4} f\left(\eta_{b}\right) \frac{G m M}{k T r_{0}^{2}} .
$$

Substituting $T_{d}=400 \mathrm{~K}, T_{n}=90 \mathrm{~K}$ into $f\left(\eta_{b}\right)$

$$
f\left(\eta_{T}\right)=\frac{2}{\sqrt{\pi}} e^{-\eta_{T}^{2}} \eta_{T}+1-\operatorname{erf}\left(\eta_{T}\right),
$$

$$
\begin{gathered}
\eta_{T}=v_{e} / \sqrt{\frac{2 k T}{m}}=\sqrt{\frac{2 G M}{r_{0}}} / \sqrt{\frac{2 k T}{m}}, \\
v_{e}=2.38 \mathrm{~km} / \mathrm{s}, \quad \sqrt{\frac{2 k T_{d}}{m}}=1.28 \mathrm{~km} / \mathrm{s}, \\
\sqrt{\frac{2 k T_{n}}{m}}=0.609 \mathrm{~km} / \mathrm{s},
\end{gathered}
$$

$\eta_{T}$ denotes $\eta_{b}(T), \eta_{d}$ and $\eta_{n}$ denote $\eta_{b}\left(T_{d}\right)$ and $\eta_{b}\left(T_{n}\right)$ respectively.

$\eta_{d}=1.86, \eta_{n}=3.91$.

$$
\begin{aligned}
f\left(\eta_{d}\right) & =0.0744, \\
f\left(\eta_{n}\right) & =1.04 \times 10^{-6}, \\
\bar{v}_{d} & =\frac{2}{\sqrt{\pi}} \sqrt{\frac{2 k T_{d}}{m}}=1.45 \mathrm{~km} / \mathrm{s}, \\
\bar{v}_{n} & =\frac{2}{\sqrt{\pi}} \sqrt{\frac{2 k T_{n}}{m}}=0.688 \mathrm{~km} / \mathrm{s} .
\end{aligned}
$$

Substituting this into (3B), one obtains:

$$
\left\{\begin{array}{l}
\lambda_{d}=5.31 \times 10^{-5} \mathrm{~s}^{-1}, \\
\lambda_{n}=3.52 \times 10^{-10} \mathrm{~s}^{-1},
\end{array}\right.
$$

and the equivalent decay constant for uranium and thorium series is

$$
\lambda_{r} \sim\left(10^{-10}-10^{-9}\right) y^{-1} \approx\left(10^{-16}-10^{-17}\right) \mathrm{s}^{-1} .
$$

Therefore,

$$
\lambda_{d} \gg \lambda_{n} \gg \lambda_{r}
$$

It means the escape rate during daytime is much greater (about 5 orders of magnitude) compared to the escape rate during night, and the escape rate of helium gas on the surface of the Moon at day or night is much greater (about 7 to 12 magnitudes) than the production rate of helium gas due to the emission of alpha particles by uranium and thorium series nuclides. In this case,

$$
\lambda_{n} \pm \lambda_{r} \sim \lambda_{n} \quad \text { and } \quad \lambda_{d} \pm \lambda \sim \lambda_{d} .
$$

Now we proceed to solve the set of equations $(4 \mathrm{~A})-(4 \mathrm{C})$

We choose a suitable time $t_{0}$ as a starting point of day, regarding $t_{0}$ to $t_{0}+(R / 2)$ as day and $t_{0}+(R / 2)$ to $t_{0}+R$ as night, $R$ being the rotation period of Moon. As $t-t_{0} \rightarrow t$, assuming the initial condition 


$$
N=N_{0}, \quad N_{r}=N_{r 0}, \quad \text { at } t=0,
$$

the solution of $(4 \mathrm{~A})-(4 \mathrm{C})$ satisfying (6) is:

$$
\begin{aligned}
N_{r}(t)= & N_{r 0} e^{-\lambda_{r} t}, \\
N(t)= & \left(N_{0}-\frac{\lambda_{r}}{\lambda_{d}-\lambda_{r}} N_{r 0}\right) e^{-\lambda_{d} t} \\
& +\frac{\lambda_{r}}{\lambda_{d}-\lambda_{r}} N_{r 0} e^{-\lambda_{r} t}, \quad 0 \leq t \leq \frac{R}{2} \text { (day), } \\
N(t)= & \left(N\left(\frac{R}{2}\right)-\frac{\lambda_{r}}{\lambda_{n}-\lambda_{r}} N_{r 0} e^{-\lambda_{r} \frac{R}{2}}\right) e^{-\lambda_{n}\left(t-\frac{R}{2}\right)} \\
& +\frac{\lambda_{r}}{\lambda_{n}-\lambda_{r}} N_{r 0} e^{-\lambda_{r} t}, \quad \frac{R}{2}<t \leq R \text { (night). }
\end{aligned}
$$

Here $N(R / 2)$ in (9) is determined by (8).

If $\lambda_{r} t \ll 1, e^{-\lambda_{r} t} \approx 1 \pm \lambda_{r} \approx 1$ or $N_{r}(r)=N_{r 0}$. For example, if $t=100 y, e^{-\lambda_{r} t} \approx 1-10^{-7} \approx 1$.

If $t=10^{4} y, e^{-\lambda_{r} t} \approx 1-10^{-3}$, the error is still within $10^{-3}$. In the above approximation and (5A),

$$
N\left(\frac{R}{2}\right)=N_{0} e^{-\lambda_{d} \frac{R}{2}}+\frac{\lambda_{r} N_{r 0}}{\lambda_{d}}\left(1-e^{-\lambda_{d} \frac{R}{2}}\right) .
$$

From equations (9) and (10) one obtains

$$
\begin{aligned}
N(R)= & N_{0} e^{-\left(\lambda_{d}+\lambda_{n}\right) \frac{R}{2}}+\lambda_{r} N_{r 0} \\
& \times\left[\frac{1}{\lambda_{d}} e^{-\lambda_{n} \frac{R}{2}}\left(1-e^{-\lambda_{d} \frac{R}{2}}\right)+\frac{1}{\lambda_{n}}\left(1-e^{-\lambda_{n} \frac{R}{2}}\right)\right] .
\end{aligned}
$$

In the steady state case, the solution is different for day and night, such a solution is just a periodical solution with $R$, divided between day $(R / 2)$ and night $(R / 2)$. In order to get a periodical solution with period $R$, we require:

$$
N(R)=N_{0} .
$$

From (11) and (12) one obtains:

$$
\begin{aligned}
N(R)= & N_{0}=\frac{\lambda_{r} N_{r 0}}{1-e^{-\left(\lambda_{d}+\lambda_{n}\right) \frac{R}{2}}} \\
& \times\left[\frac{1}{\lambda_{d}} e^{-\lambda_{n} \frac{R}{2}}\left(1-e^{-\lambda_{d} \frac{R}{2}}\right)+\frac{1}{\lambda_{n}}\left(1-e^{-\lambda_{n} \frac{R}{2}}\right)\right] .
\end{aligned}
$$

Under the requirement of periodical solution (12) we integrate $(4 \mathrm{~B})$

$$
\int_{0}^{R / 2} \frac{d N}{d t} d t=\int_{0}^{R / 2} \lambda_{r} N_{r}(t) d t-\lambda_{d} \int_{0}^{R / 2} N(t) d t .
$$

The result is

$$
\begin{aligned}
N\left(\frac{R}{2}\right)-N_{0} & =\lambda_{r} N_{r 0} \frac{R}{2}-\lambda_{d} \bar{N}_{d} \frac{R}{2}, \\
\bar{N}_{d} & \equiv \frac{1}{R / 2} \int_{0}^{R / 2} N(t) d t .
\end{aligned}
$$

Similarly, we integrate (4C) from $R / 2 \rightarrow R$, and obtain

$$
\begin{aligned}
N(R)-N\left(\frac{R}{2}\right) & =\lambda_{r} N_{r 0} \frac{R}{2}-\lambda_{n} \bar{N}_{n} \frac{R}{2}, \\
\bar{N}_{n} & \equiv \frac{1}{R / 2} \int_{R / 2}^{R} N(t) d t,
\end{aligned}
$$

$\bar{N}_{d}$ and $\bar{N}_{n}$ denote the mean total number of molecules during day and night respectively.

Let

$$
\Delta N(R / 2) \equiv N_{0}-N\left(\frac{R}{2}\right)
$$

Since $\lambda_{d}>\lambda_{n}, \quad \therefore \Delta N(R / 2)>0$.

From the condition (12) $N(R)=N_{0}$, and from (14) and (15) we have

$$
\begin{aligned}
\frac{\lambda_{d} \bar{N}_{d}}{\lambda_{n} \bar{N}_{d}} & =x, \\
x & \equiv \frac{\lambda_{r} N_{r 0}+\Delta N(R / 2) /(R / 2)}{\lambda_{r} N_{r 0}-\Delta N(R / 2) /(R / 2)}, \\
x & >1, \text { due to } \Delta N(R / 2)>0 .
\end{aligned}
$$

The relation between total atmospheric molecular number $N$ and the molecular number density on the surface $n_{0}$ is $N=4 \pi r_{0}^{2} n_{0} \mathrm{kT} / \mathrm{mg}$. Since the number densities on the lunar surface during day and night are $n_{0 d}$ and $n_{0 n}$ respectively, we have

$$
\frac{n_{0 d}}{n_{0 n}}=\frac{T_{n} \bar{N}_{d}}{T_{d} \bar{N}_{n}}=x \frac{\lambda_{n}}{\lambda_{d}} \frac{T_{n}}{T_{d}} .
$$


In order to calculate $x$, we begin with $\Delta N(R / 2)$. $N_{0}$ has been given in (13) from which one can obtain the expression of $N(R / 2)$ by substituting (13) into (10). By subtraction of these two terms we have

$$
\begin{aligned}
\Delta N & \left(\frac{R}{2}\right) \\
& =N_{0}-N\left(\frac{R}{2}\right) \\
& =\frac{\lambda_{r} N_{r_{0}}\left(1-e^{-\lambda_{d} \frac{R}{2}}\right)\left(1-e^{-\lambda_{n} \frac{R}{2}}\right)\left(\frac{1}{\lambda_{n}}-\frac{1}{\lambda_{d}}\right)}{1-e^{-\left(\lambda_{n}+\lambda_{d}\right) \frac{R}{2}}} .
\end{aligned}
$$

Since $\lambda_{n}$ is much less than $\lambda_{d}$, we expand $\Delta N$ into series of $\lambda_{n} R / 2$ and keeping only the linear term, one obtains

$$
\begin{aligned}
x= & \frac{\lambda_{d}}{\lambda_{n}} \frac{2}{1+\left(\lambda_{d} R / 2\right) /\left[1-\exp \left(-\lambda_{d} R / 2\right)\right]}, \\
& \text { for } \lambda_{d} \gg \lambda_{n}, \lambda_{d} R \sim 1 .
\end{aligned}
$$

Substituting into (18), we have

$$
\begin{aligned}
\frac{n_{0 d}}{n_{0 n}} & =y \frac{T_{n}}{T_{d}}, \\
y & =\frac{2}{1+\left(\lambda_{d} R / 2\right) /\left[1-\exp \left(-\lambda_{d} R / 2\right)\right]},
\end{aligned}
$$

$R$ is taken as the synodic month, that is $R=29.53$ earth days

$$
\begin{aligned}
\lambda_{d} R / 2 & =67.8, \quad y=0.0291 \\
\frac{n_{0 d}}{n_{0 n}} & =0.0291 \frac{T_{n}}{T_{d}}=0.00654 .
\end{aligned}
$$

But the measured value is $\left(n_{0 d} / n_{0 n}\right)_{\text {measure }}=$ $\left(3 \times 10^{9}\right) / 6 \times 10^{10}=0.05$.

We find that the calculated value is less than the measured ratio by one order of magnitude. The problem originated from too many molecules escaping during the lunar day, leading to the very small density ratio between day and night. Thus we have to consider other types of gas molecules, in addition to helium like Ar and Rn which can hardly escape. These molecules have heavier masses and therefore $v_{p}$ values are small, resulting in a large $\eta$, and small $f(\eta)$, finally making $\lambda$ small. For example, from (3B),

$$
\lambda_{d}(\mathrm{Ar})=2.76 \times 10^{-13} \mathrm{~s}^{-1} \ll \lambda_{n}(\mathrm{He}) .
$$

These molecules can be expected not to escape from the Moon. Then the density ratio between day and night should obey

$$
\frac{n_{0 d}(B)}{n_{0 n}(B)}=\frac{T_{n}}{T_{d}}
$$

Here $n_{0}(B)$ denotes the number density of such heavy molecules. Now we proceed to calculate their percentage abundance in the atmosphere. Suppose the density of helium is $n_{0 d}(\mathrm{He})$ and $n_{0 n}(\mathrm{He})$ during day and night respectively, from (21), we get

$$
n_{0 d}(\mathrm{He})=y \frac{T_{n}}{T_{d}} n_{0 n}(\mathrm{He}) .
$$

Suppose the number density of heavier molecules at nighttime is $n_{0 n}(B)$

$$
n_{0 n}(B)=\beta n_{0 n}(\mathrm{He}) .
$$

Here $\beta$ is the ratio of $n_{0 n}(B) / n_{0 n}(\mathrm{He})$ which remains to be determined. We denote the number density of heavier molecules at day time as $n_{0 d}(B)$, which can be obtained from (22)

$$
n_{0 d}(B)=\frac{T_{n}}{T_{d}} n_{0 n}(B) .
$$

We determine $\beta$ as follows

$$
\begin{gathered}
\left\{\begin{array}{l}
n_{0 d}=n_{0 d}(\mathrm{He})+n_{0 d}(B), \\
n_{0 n}=n_{0 n}(\mathrm{He})+n_{0 n}(B),
\end{array}\right. \\
\frac{n_{0 d}}{n_{0 n}}=\frac{(y+\beta) T_{n} / T_{d}}{1+\beta}, \\
\therefore \quad \beta=\frac{\left\{\left(\frac{n_{0 d}}{n_{0 n}}\right)-y\left(\frac{T_{n}}{T_{d}}\right)\right\}}{\left\{\left(\frac{T_{n}}{T_{d}}\right)-\left(\frac{n_{0 d}}{n_{0 n}}\right)\right\}} .
\end{gathered}
$$

Substituting $n_{0 d} / n_{0 n}=1 / 20, T_{n} / T_{d}=90 / 400, y=$ 0.0291 into (28), we have

$$
\beta=0.248
$$

This result shows that the number density of helium at night is about $75 \%$ and other heavier molecules constitute $\sim 25 \%$.

One can also evaluate the gas emission rate on the Moon 


$$
\begin{aligned}
\lambda_{r} N_{r 0} & =\frac{1}{2}\left(\lambda_{d} \bar{N}_{d}+\lambda_{n} \bar{N}_{n}\right) \approx \frac{1}{2} \lambda_{d} \bar{N}_{d} \\
& =\frac{1}{2} \pi r_{0}^{2} \bar{v}\left(T_{d}\right) f\left(\eta_{d}\right) n_{0 d}(\mathrm{He}) .
\end{aligned}
$$

From (23)-(26)

$$
n_{0 d}(\mathrm{He})=y \frac{T_{n}}{T_{d}} \cdot \frac{n_{0 n}}{1+\beta} .
$$

Substituting $y=0.0291, T_{n} / T_{d}=90 / 400, \quad \beta=$ $0.248, n_{0 n}=6 \times 10^{10}, r_{0}=1.738 \times 10^{6} \mathrm{~m}, \bar{v}\left(T_{d}\right)=$ $1.45 \times 10^{3} \mathrm{~m} / \mathrm{s}, f\left(\eta_{d}\right)=0.0744$ and (30) into (28), one obtains

$$
\lambda_{r} N_{r 0}=1.61 \times 10^{23} \mathrm{~s}^{-1} .
$$

This indicates that the amount of helium emitted is about $1 \mathrm{~g}$ per second and

$$
N_{r 0} \sim \frac{1}{\lambda_{r}} \times 1.61 \times 10^{23} \mathrm{~s}^{-1} .
$$

Taking $\lambda_{r} \sim\left(10^{-16}-10^{-17}\right) \mathrm{s}^{-1}$

$$
N_{r 0} \sim\left(1.61 \times 10^{39}-1.6 \times 10^{40}\right) .
$$

Of course, it does not correspond to the total number of thorium and uranium atoms on the Moon, since in the decay processes one thorium or uranium atom emits 5 to $12 \alpha$ particles and then decays into a stable element whereas in the above calculation, only one $\alpha$ particle was considered. Therefore, the total number of thorium or uranium atoms should be revised as $(1 / 5)-(1 / 12) N_{r 0} \sim$ $(1 / 8) N_{r 0}$, corresponding to

$$
\begin{aligned}
M_{r} \sim 1.6 \times 240 \times 1.674 \times 10^{-27} \times\left(10^{39} \sim 10^{40}\right) \\
\quad \times \frac{1}{8} \mathrm{~kg} \sim 8.1 \times\left(10^{13} \sim 10^{14}\right) \mathrm{kg} .
\end{aligned}
$$

This amount is equivalent to $1.1 \times\left(10^{-9} \sim 10^{-8}\right)$ the mass of Moon.

It should be pointed out that in the above calculations, we did not consider the solar wind and cosmic ray implanted ions which may escape during day time in the lunar environment. The main ingredients of solar wind are protons. The average velocity of the solar wind is $v=\sim 450 \mathrm{~km} / \mathrm{s}$ and the number density of solar wind is $8 \times 10^{6} \mathrm{~m}^{-3}$. From the mean speed of the proton $v=450 \mathrm{~km} / \mathrm{s}$, the energy is calculated to be about $E_{p} \sim 2.1 \times 10^{-3} \mathrm{Mev}$. This energy is too small to induce nuclear reactions and can only produce elastic or inelastic scattering. A fraction of the protons scattered in space immediately escapes since the velocity is far more than the escape velocity on the surface of the Moon $(\sim 2.4 \mathrm{~km} / \mathrm{s})$. A fraction of them are also absorbed by the lunar soil or rocks due to multi-inelastic-scattering and others lose their energy to form the atmosphere on the Moon with Maxwellian distribution. Suppose each solar wind proton captures an electron to form a hydrogen atom, which subsequently forms hydrogen molecules then, formula (3A) can be used to estimate the escape rate of hydrogen molecules.

From formula (3B)

$$
\begin{aligned}
\eta_{d}\left(\mathrm{H}_{2}\right) & =\sqrt{\frac{2 G M}{r_{0}}} / \sqrt{\frac{2 k T}{m\left(\mathrm{H}_{2}\right)}} \approx 1.32, \\
\eta_{d}(\mathrm{H}) & \approx 0.93 \\
f_{H_{2}}\left(\eta_{d}\right) & \approx 0.324 \\
f_{H}\left(\eta_{d}\right) & \approx 0.632, \\
\lambda_{d}\left(\mathrm{H}_{2}\right) & \approx 1.64 \times 10^{-4} \mathrm{~s}^{-1}, \\
\lambda_{d}(\mathrm{H}) & \approx 2.26 \times 10^{-4} \mathrm{~s}^{-1} .
\end{aligned}
$$

Then, the variation of the total hydrogen number $N$ is

$$
\frac{d N}{d t}=J-\lambda_{d} N
$$

Here $J$ is the flux of incident solar wind and $\lambda_{d} N$ is the escape rate on the surface of the Moon,

$$
J=\pi r_{0}^{2} v n_{s}, \quad N=4 \pi r_{0}^{2} k T n_{d} / \mathrm{mg} .
$$

Here $n_{s}=8 \times 10^{6} \mathrm{~m}^{-3}$ is the number density of solar wind and $n_{d}$ is the number density of hydrogen. In steady state case, $d N / d t=0$.

Then, we have

$$
J=\lambda_{d} N \quad \text { or } \quad n_{d}=\left(m g v / 4 \mathrm{kT} \lambda_{d}\right) n_{s} .
$$

Then, we have

$n_{d}\left(\mathrm{H}_{2}\right) \sim 5.4 \times 10^{9} \mathrm{~m}^{-3}, \quad n_{d}(\mathrm{H}) \sim 2.8 \times 10^{9} \mathrm{~m}^{-3}$.

This is an overestimate of the hydrogen number density since a large fraction of the scattered protons are absorbed by the lunar soil and rocks or escape immediately to space and only a small fraction becomes a part of the lunar atmosphere. Even so the solar wind contributes a significant factor to the number density of the lunar atmosphere. 


\section{Conclusions}

Based on the above calculations we can draw the following conclusions:

- Assuming that there existed an atmosphere of $1 \mathrm{~atm}$ on the Moon, with temperature below $240 \mathrm{~K}$, the atmosphere would then be retained and a very small fraction of molecules would escape.

- If there existed an atmosphere on the Moon in the beginning and its temperature distribution was similar to the current temperature distribution on the Earth, then escape time is calculated to be $\tau=1.8 \times 10^{6} \mathrm{y}$, and the atmosphere would have completely escaped a long time ago.

- Based on the ratio of the measured number density between day and night, it is estimated that the tenuous atmosphere on the Moon is composed of $75 \% \mathrm{He}$ and $25 \%$ Ar. The gas emission rate on the surface of the Moon is about $1.61 \times 10^{23} \mathrm{~s}^{-1}$, equivalent to the emission of $1 \mathrm{~g}$ of helium per second assuming dynamic equilibrium between emission and escape.

- Solar wind contributes a non-negligible fraction in the number density of the lunar atmosphere.

\section{Acknowledgements}

We are thankful to the reviewers for many precious and helpful comments.

\section{References}

Da Dao-an and Yang Ya-tian 2005 Life time of atmospheres on Earth and Venus; Vacuum and Cryogenics 11(2) $70-77$.

Konpaneetz A S 1957 Theoretical Physics; Gostechizdat, 2nd Edition. 